\title{
Maximising uptake and utilization of molecular diagnostics: lessons from implementation evaluation of tuberculosis diagnostics
}

Nyanda Elias Ntinginya

National Institute of Medical Research-Mbeya Medical Research Centre

Fred Orina

Kenya Medical Research Institute

Ivan Mwebaza

Makerere University

Alphonce Liyoyo

Institute of Clinical Research Institute

\section{Barbara Miheso}

Kenya Medical Research Institute

\section{Augustus Aturinde}

Lund University: Lunds Universitet and Kyambogo University

\section{Fred Njeleka}

National Institute of Medical Research-Mbeya Medical Research Centre

\section{Fred Njeleka}

National Institute of Medical Research-Mbeya Medical Research Centre

\section{Elizabeth F Msoka}

Kilimanjaro Clinical Research Institute

\section{Helen Meme}

Kenya Medical Research Institute

\section{Erica Sanga}

National Institute for Medical Research-Mbeya and Mwanza Research Centres

\section{Erica Sanga}

National Institute for Medical Research-Mbeya and Mwanza Research Centres

\section{Davis Kuchaka}

Kilimanjaro Clinical Research Institute

\section{Simeon Mwanyonga}

National Institute for Medical Research - Mbeya Medical Research Centre

\section{Willyhelmina Olomi}

National Institute for Medical Research - Mbeya Medical Research Centre 
Kilimanjaro Clinical Research Institute

\section{Linda Minja}

Kilimanjaro Clinical Research Institute

\section{Moses Joloba}

Makerere University

\section{Blandina Theophil Mmbaga}

Kilimanjaro Clinical Research Institute

\section{Evans Amukoye}

Kenya Medical Research Institute

\section{Stephen H Gillespie}

University of St Andrews

\section{Wilber Sabiiti ( $\sim$ ws31@st-andrews.ac.uk )}

The University of St Andrews https://orcid.org/0000-0002-4742-2791

\section{Research}

Keywords: Implementation, Molecular diagnostics, Tuberculosis, Maximising uptake and utilization, policy and practice.

Posted Date: November 13th, 2020

DOI: https://doi.org/10.21203/rs.3.rs-106037/v1

License: (c) (i) This work is licensed under a Creative Commons Attribution 4.0 International License. Read Full License 


\section{Abstract}

Background: Tuberculosis (TB), a treatable disease claims over a million lives every year. Accurate rapid diagnosis is crucial for early treatment initiation and prevention of severe disease. Despite over 10 years approval of molecular diagnostics for routine use, an estimated 3 million TB cases go undetected per year. We investigated the barriers and opportunities to maximise uptake and utilization of molecular diagnostics in routine healthcare settings.

Methods: We deployed surveys, healthcare facility audits, focus group discussions, in-depth interviews, and policymaker dialogues to unravel factors affecting the uptake and utilization of TB molecular diagnostics in three East African countries. The benchmark was the World Health Organization approved Xpert MTB/RIF and Line Probe Assay (LPA) implemetation at district and regional hospital level respectively.

Results: 190 district and county health officers participated in the survey. The survey findings were corroborated by 145 healthcare facility (HCF) audits and 11 policymaker engagement workshops. At $66 \%$ coverage, Xpert MTB/RIF fell behind microscopy and clinical diagnosis by $33 \%$ and $1 \%$ respectively across 190 districts/counties. Stratified by HCF type, Xpert MTB/RIF implementation was $56 \%, 96 \%$ and $95 \%$ at district-, regional- and national referral- hospital level. LPA coverage was $4 \%, 3 \%$ below culture across the three countries. Out of 111 HCFs with Xpert MTB/RIF, 37 (33\%) utilized it to full capacity, performing $\geq 8$ tests per day of which $51 \%$ of these were level five (zonal consultant and national referral) HCFs. Likewise, $75 \%$ of LPA test performance was at level five HCFs. Underutilization of Xpert MTB/RIF and LPA was mainly attributed to inadequate- human resource, $22 \%$ and utilities, $26 \%$ respectively. Absence of the diagnostic services was attributed to under financing. Lack of awareness was second to underfinancing as reason underlying absence of LPA service. Creation of a health tax and decentralising collection and management of this tax to district/county level was proposed by policymakers as means to boost domestic financing for uptake of health technologies.

Conclusion Our findings show higher uptake and utilization of molecular and other diagnostics at tertiarythan primary-secondary- level HCFs. Innovative implementation models to ensure quality access at lower level HCFs are urgently needed.

\section{Contributions To Literature}

- This study shows implementation of molecular diagnostics is higher at tertiary healthcare level contrary to World Health Organization recommendation to have them at lower healthcare level where they could be accessed by the majority.

- It demonstrates that utilization of molecular- and culture- based diagnostics that require advanced laboratory facilities increases the higher you go in the health system hierarchy, which implies low access by people living farther afield. 
- The study shows that it is not only the unavailability of finances that drives poor implementation but also mechanisms by which the finances are invested and priorities set.

\section{Introduction}

The COVID-19 pandemic led to world-wide shutdown and seen unprecedented uptake and utilization of molecular diagnostics with over 500 million tests conducted in a period of 9 months (statista.com Oct 9th 2020). The rapidly evolving COVID-19 is responsible 36.99 million cases and 1.1 million deaths (WHO COVID-19 Dashboard 11th Oct 2020)(1). The health systems have been shaken but most importantly the pandemic has raised public consciousness of the value of diagnostics and interdisciplinary approaches in the control of diseases. In contrast, tuberculosis (TB) has been a pandemic for time immemorial and a global public health emergency for over 20 years $(2,3)$. A quarter (1.7 billion) of the world population have TB infection and in 2018, 10 million developed active disease resulting in 1.5 million deaths(4-6). Of the 10 million notified cases, $55 \%$ were bacteriologically confirmed and a small proportion of these were tested using rapid molecular tests(5). The rate of TB testing does not match incidence of TB disease and, consequently, an estimated 3 million cases go undetected every year(7).

WHO approved routine use of TB molecular tests more than 10 years ago, starting with the Line Probe Assay (LPA) in 2008 and Xpert MTB/RIF in 2010 $(8,9)$. The latter detects both TB and resistance to rifampicin in 2 hours and is the widely used molecular test for TB. By 2016, only 16 million Xpert MTB/RIF tests had been performed, translating into 3.2 million tests per year(10). Whilst there is been steady progress in the uptake and use of Xpert MTB/RIF, the impact of using the same GeneXpert platform for testing HIV viral load and more recently COVID-19 on TB diagnosis is yet to be unravelled(11, 12). There is sharp contrast in the rate of uptake of COVID-19 molecular tests compared to TB and could a leaf be borrowed from either disease on how to accelerate and maximise translation of health research innovations into policy and practice.

The impact of effective diagnostic testing is not only the ability to detect disease but also contribution to favourable clinical outcomes for patients. This is more likely to be achieved in a health system landscape that employs holistic approach to patient care as enshrined in the "WHO End TB strategy": integrated patient-centred care and prevention to foster early diagnosis, universal drug-susceptibility testing, systematic screening of contacts and high-risk group, treatment of all people. Good governance and leadership to ensure equal access to healthcare, safe medicines and social protection to minimise impact of economic status on quality of care received. Last but not least, intensifying research and innovation to ensure discovery, development, optimisation and rapid uptake of new tools, interventions and strategies coupled with monitoring and evaluation to ascertain impact(13-15).

Low- and Middle- Income Countries (LMICs) account for over 50\% of the global TB burden(5). The scarcity of resources complicates the development of strong health systems and more so the COVID-19 effect which is estimated to have reduced TB care delivery by $20 \%$ is taken into account(12). Models to ensure access of service by those that need them the most have been developed(16-18). For instance, 
Kenya recently used Patient Pathway Analysis (PPA) model to assess the delivery of TB diagnostic and treatment and found more than $50 \%$ of the low-level healthcare facilities (HCFs) where majority people seek care did not have diagnostic services(19). Confronted by this finding, Kenya committed to redesigning the provision of TB services to ensure they are in places where they are most needed rather than where it is operationally convenient, such as tertiary level healthcare facilities (HCFs) in urban settings. Kenya adopted this approach around the same time as our study, it will be interesting to assess the impact made on uptake and utilization of molecular diagnostics for TB in the country.

Using the implementation of TB diagnostics as a model, we sought to understand the ways to maximise uptake and utilization of diagnostic technologies that have regulatory approval and are supported by WHO and other expert opinion within the health systems. Our findings show higher rate of uptake and utilization of TB diagnostics at tertiary level HCFs. In contrast to the WHO endorsement, LPA is mainly implemented at zonal - national level instead of regional HCF while Xpert MTB/RIF was more utilised at regional, zonal - national level HCFs instead of the recommended district level hospitals. These data point to hub-centralised model of implementation in order to maximise both uptake and utilization of the diagnostics platforms. Resolving the health system, socioeconomic and cultural bottlenecks is pivotal for the effective implementation of either the PPA or centralised model of diagnostic services.

\section{Methods}

This was a mixed methods study employing both quantitative and qualitative approaches including surveys of district/county health officers, healthcare facility audits, in-depth one-on-one interviews, focus group discussions and dialogues with policymakers. The study was conducted between Sept 2016 to Dec 2017 in Uganda, Kenya and Tanzania. At the time of the study, only Kenya was defined as a middle income country, and it has now been joined by Tanzania in this classification(20).

\section{Consortium}

The study was conducted under the TWENDE consortium and comprised 7 institutions: Makerere University and CPAR Uganda Ltd Uganda, Kenya Medical Research Institute (KEMRI) Kenya, Kilimanjaro Clinical Research Institute (KCRI) and National Institute of Medical Research - Mbeya Medical Research Centre (NIMR-MMRC) Tanzania while the East African Community was represented by the East African Health Research Commission (EAHRC) and University of St Andrews, UK. TWENDE, which is Swahili word means "let's go!" is abbreviated from Tuberculosis: Working to Empower the Nations' Diagnostic Effort.

\section{Geography and participants}

Areas of coverage were selected based on the TB diagnostics, Line Probe Assay (LPA) and Xpert MTB/RIF (GeneXpert) approved areas of implementation. According to the World Health Organisation, LPA and Xpert MTB/RIF are recommended for implementation at regional and district level hospitals respectively. In Uganda, regions were based on areas represented by a regional hospital whilst districts were taken as they are currently structured as administration units. In Tanzania, regions and districts are clearly demarcated administrative units and so were covered as such. For Kenya, regional units were 
counties whilst the sub-counties were equivalent to districts in Uganda and Tanzania. Study districts, regions or counties were purposively selected to be representative of all geographical regions of the country.

Participants were healthcare administrators such as regional/county and district health (medical) officers, HCF managers and healthcare professionals of participating HCFs. Apart from managers and practitioners, patients, TB survivors, Community health Volunteers, opinions leaders among the HCF users, local council leaders, and national policy makers in ministry of Health and parliament were engaged. Participants were selected based on their role either as healthcare leader/manager or practitioner and/or by their status as TB patients, caregivers or survivors. The managers and practitioners represented opinion from health system service delivery perspective whilst the patients, caregivers and survivors shared their lived experiences in accessing diagnosis and treatment as well as living with TB in a community.

\section{Survey}

An online survey was constructed using the University of St Andrews licenced Qualtrics survey tool (https://standrews.eu.qualtrics.com). The tool was commissioned by the University of St Andrews teaching and research ethics and complies with the European Union General Data Protection regulation (GDPR) EU2016/679 and the local country Ethics committees. The purpose of the survey was to obtain an overview of the challenges and opportunities confronting tuberculosis diagnostics and treatment services from the perspective of regional/county and district/sub-county health or medical officers (see Additional file 1: Survey Questionnaire). It sought to know what facility and/or service is available and if not available, what could be the underlying reasons. The survey was made compatible with all computer systems, Windows and macOS, and both Android and Apple smart phones. The opening page of the questionnaire had participant information and solicitation for consent to participate in the study.

\section{Healthcare facility audits}

The rationale of the audit was to verify the responses given by survey participants and gain more insight into the implementation of TB diagnostics on ground. The audit tool was developed and teams of researchers in each country were trained to administer the tool at selected healthcare facilities. The respondents at the HCFs were the healthcare and laboratory managers at regional/county and district/sub-county hospitals. The audit ascertained whether the facilities and services reported by the survey participants were indeed available and being utilized. In addition to interviews with healthcare practitioners, the auditors inspected the facilities to verify answers given. See Additional file 2: the Audit tool.

\section{Policy dialogues}

Workshops were organised targeting policy makers and implementers to discuss views from the general community and construct actionable policy briefs for the national policies to implement. Participants in these were workshops were parliamentarians, technocrats from Ministries of Health, leaders from regional/county administrative units, and representatives of other disease control agencies. 
"Chatham House Rules" were used during the FGDs and policy dialogues to promote open expression of views and opinions without fear of being vilified. The Chatham rule states: "When a meeting, or part thereof, is held under the Chatham House Rule, participants are free to use the information received, but neither the identity nor the affiliation of the speaker(s), nor that of any other participant, may be revealed".

\section{Data Analysis}

Quantitative data was analysed using Microsoft Excel 2016 and GraphPad Prism v.6. Percentage diagnostic coverage and utilization was calculated per type of HCF and all HCFs combined. ANOVA multiple comparisons test was used to assess the performance difference between HCFs. Significance was considered at $p<0.05$ and $95 \%$ confidence interval $(\mathrm{Cl})$.

\section{Results}

\section{Participants and study area}

A total of 217 survey responses received from district or county healthcare officers across the three countries. After removal of duplicate/multiple responses per district or county, 190 entries representing 27 (14\%), 66 (35\%) and 97 (51\%) from Kenyan counties, Tanzanian and Ugandan districts respectively were considered for analysis. The Kenyan county was considered equivalent of Ugandan or Tanzanian region. The 27 Kenyan counties varied in size, median (range) 5 (2-17) sub-counties (districts), together constituting an area of 159 sub-counties and $57 \%$ of Kenyan counties. All the 14 Ugandan and 26 Tanzania mainland regions were represented in the survey. Time spent in the post by respondents ranged from less than one year to 16 years: 14 (7\%) less than one year, $35(16 \%)$ one to two years, $53(24 \%)$ three to four years, 41 (19\%) five to six years, $29(13 \%)$ seven to eight years, $16(7 \%)$ nine to ten years and 29 (13\%) over10 years.

The on-site audits healthcare facilities (HCFs) recapitulated the survey results. A total of $145 \mathrm{HCFs}$ were audited, 48 (33\%) each for Kenya and Uganda and 49 (34\%) Tanzania. This represents 42\% (112), 29\% (169) and 44\% (47) of the districts and counties in Uganda, Tanzania and Kenya respectively at the time of audit. In all the countries, government was the main healthcare provider, owning over $80 \%$ of the healthcare facilities. Level 5 hospitals (national, consultant level, regional referral) were 42, level 4 (regional hospitals) 34, level 3 (district hospitals) 57, level 2 (health centre IV) 6 and level 1 (dispensary, health centre 1-3) 6 . The majority of the tuberculosis laboratories were able to deliver Biosafety level II (BSL II), 86 followed by BSL I, 23 and BSL III, 6 and 4 general laboratories.

\section{Coverage of diagnostics}

At the time of the survey; smear microscopy was the most available diagnostic tool, $97 \%(185 / 190)$ followed by clinical diagnosis, 67\% (128/190) and Xpert MTB RIF, 66\% (125/190) of the districts/counties surveyed. Tuberculosis culture and Line Probe Assays (LPA) were the least available, 7\% (13/190) and 4\% (8/190). Most of the districts, $89 \%$ (169) reported microscopy in combination with other diagnostics leaving only $11 \%$ (21) which had only microscopy for diagnosis. At country level, Xpert MTB/RIF test 
coverage was 74\% (72/97) and 39\% (26/66) districts in Uganda and Tanzania respectively and $42 \%$ $(67 / 159)$ sub-counties in Kenya (Fig. 1).

The number of Xpert MTB/RIF test machines (GeneXpert) was not consistent with the number of subcounties (districts) per county in Kenya. Some counties had less than the number of the sub-counties and vice versa (Fig. 2).

The coverage results were replicated in the on-site audit of 145 HCFs: smear microscopy availability 142 (98\%), Xpert MTB/RIF 111 (76\%), Culture 5 (4\%) and LPA 4 (3\%). Number of available of Xpert MTB/RIF instruments increased with increasing level of HCF. Out of the $111 \mathrm{HCFs}$ possessing Xpert MTB/RIF, 1 (0.9\%), 5 (5\%), 32 (29\%), 33 (30\%) and 40 (36\%) were level 1, 2, 3, 4 and 5 HCFs respectively. Availability within the HCF type, level 4 and 5 had the highest, $97 \%$ and $95 \%$ coverage of Xpert MTB/RIF instruments followed by level 2, 83\%, level 3 (56\%) and level 1, 17\% (Fig. 3).

Out the 4 HCFs in possession of LPA, three were L5 (1 national-, 1 zonal- referral and 1 consultant hospital), and only one was L4 (regional). All the five HCFs with culture were L5 (2 national referral, 2 consultant and 1 zonal referral hospitals).

\section{Utilization of diagnostics}

\section{Smear microscopy}

Taken together, number of smears performed per month by HCFs with microscopy services (142) were median (range) 56 (0.8-460). The number of smears performed increased with the HCF level, 26 (17-75), 18 (1-3-60), $46(0 \cdot 8-460), 48(1 \cdot 3-400)$ and 89 (5-311) at HCF level 1, 2, 3, 4 and 5 respectively. Only HCF level 5 performed above median number of smears per month. Of the $142 \mathrm{HCFs}$ that performed microscopy, 96\% (137) used it for diagnosis and treatment follow-up. The most frequent use of smear microscopy was treatment follow-up, 50\% (68) followed by combination of primary diagnosis and treatment follow-up, $40 \%$ (55). The proportion of using microscopy as a primary diagnostic tool was high, $33 \%$ in level one (L1) HCFs versus $10 \%$ in L5 HCFs, and $67 \%$ versus $26 \%$ for using microscopy for both primary diagnosis and treatment follow-up (Table 1) 
Table 1

Utilization of smear microscopy in audited HCFs. There is high utilization of microscopy as a primary diagnostic tool in lower level HCFs

\begin{tabular}{|c|c|c|c|c|c|c|}
\hline & Total & $\begin{array}{l}\mathrm{L1}(\mathrm{N}= \\
6)\end{array}$ & $\begin{array}{l}\mathrm{L} 2(\mathrm{~N}= \\
6)\end{array}$ & $\begin{array}{l}\mathrm{L3}(\mathrm{N}= \\
57)\end{array}$ & $\begin{array}{l}\mathrm{L} 4(\mathrm{~N}= \\
34)\end{array}$ & $\begin{array}{l}\mathrm{L} 5(\mathrm{~N}= \\
42)\end{array}$ \\
\hline Primary diagnosis & 12 & $2(33 \%)$ & $0 \%$ & $6(11 \%)$ & $0 \%$ & $4(10 \%)$ \\
\hline $\begin{array}{l}\text { Primary diagnosis of HIV negative } \\
\text { cases }\end{array}$ & 2 & $0(0 \%)$ & $0 \%$ & $2(4 \%)$ & $0 \%$ & $4(10 \%)$ \\
\hline Primary diagnosis and Follow-Up & 55 & $3(67 \%)$ & $3(50 \%)$ & $\begin{array}{l}30 \\
(53 \%)\end{array}$ & $7(21 \%)$ & $\begin{array}{l}11 \\
(26 \%)\end{array}$ \\
\hline Follow-up only & 68 & $0(0 \%)$ & $3(50 \%)$ & $\begin{array}{l}17 \\
(30 \%)\end{array}$ & $\begin{array}{l}24 \\
(71 \%)\end{array}$ & $\begin{array}{l}24 \\
(57 \%)\end{array}$ \\
\hline
\end{tabular}

\section{Xpert MTB/RIF}

Like coverage, utilization of Xpert MTB/RIF increased with level of HCF, median (IQR) 0 (0-4), 90 (33390), 120 (55-170), 200 (110-240), 228 (110-320) tests per month at level 1, 2, 3, 4 and 5 HCF respectively, linear regression $p=0.002$. The ordinary one-way ANOVA multiple comparisons test showed significant difference of tests performed at different HCFs, $F=5 \cdot 4, p=0 \cdot 0005$. However, the difference was driven by tests at level 4 and 5 which were significantly higher than level 1 and both level 1 and 3 HCFs respectively. Out of the 111 sites with Xpert MTB/RIF test instruments, only 37 (33\%) utilized the instruments to full capacity, performing at least 8 tests per day or 240 tests per month. Of the HCFs using Xpert MTB/RIF to full capacity, 8\% (3), 14\% (5), 27\% (10) and 51\% (19) of these HCFs were level 2, 3, 4 and 5 respectively. None of the level 1 HCFs performed Xpert MTB/RIF to capacity (Fig. 4A and B).

All Xpert MTB/RIF test machines except $2(1 \cdot 8 \%)$ were operated in designated laboratory space and not at point-of-care (consultation room) or ward. Most HCFs prescribed Xpert MTB/RIF test to all presumptive TB cases, 86\% (95/111). Where Xpert MTB/RIF was not prescribed to all these groups of cases were prioritised, "health workers, prisoners, children (0-14 years), diabetic mothers, HIV positive clients who are coughing and on retreatment, contacts of drug resistant TB patients, retreatment cases, treatment absconders, relapse cases, re- treatment cases, refugees, and expecting mothers". It is important to note that by the end of 2019, Kenya, Tanzania and Uganda had updated their national TB control guidelines recommending Xpert MTB/RIF test to all presumptive cases hence more HCFs are currently having the Xpert MTB/RIF machines. Xpert MTB/RIF machine operational condition and reagent procurement were assessed and showed $87 \%(96 / 111)$ of the HCFs had their machines calibrated, $89 \%$ (99/111) had stable supply of cartridges and $76 \%(85 / 111)$ received the cartridges on time. The magnitude of effect by each factor varied from HCF-to-HCF with a trend to better service at high HCF level (Fig. 5A-F).

\section{Line Probe Assay (LPA)}


Utilization of the LPA was quite irregular. Out of the $4 \mathrm{HCF}$ s that had an established LPA service, one performed it on request (64 tests per month), the 2 nd was twice a month (24 tests per month) and the 3rd performed only the day they were trained and since then never performed it again. When asked what the limiting factor was for not performing LPA again, they said, "can't tell, a team came here trained us on LPA and left. since then we have never done anything, we actually don't remember what we learnt" Laboratory manager at a Regional Hospital. The fourth HCF gave no answer on how they utilized their LPA service. Of the 4 HCFs that had LPA, 3 (75\%) were level 5 and 1 (25\%) was level 4.

\section{Underlying limitations for underutilization or absence of Xpert MTB/RIF and LPA services}

Some HCFs that had Xpert MTB/RIF attributed underutilization of the service to inadequate human resource $22 \%$ (24/111), procurement difficulties, $12 \%$ (13/111), poor electricity- and water- supply, $12 \%$ $(24 / 111)$ and $6 \%(7 / 111)$ respectively. The lack of Xpert MTB/RIF and LPA services was mainly attributed to insufficient finance at both district/county level (91\% and 55\%), and at the HCF level (56\% and 21\%) respectively. Lack of awareness was a second limiting factor to financing revealing $33 \%$ and $49 \%$ of the district/county health officers and healthcare practitioners were unware of the LPA as a diagnostic test for TB. Inadequate human resource (24\%), lack of water (26\%) and electricity (26\%) were also considered substantial limitation of implementation of Xpert MTB/RIF by practitioners at their HCFs (Table 2). 6-9\% reported procurement difficulties for the two diagnostics, which included reagent stockouts and delayed payment of requisitions by the healthcare management. "Failed procurement is not necessarily due to distant (overseas) sources of laboratory supplies, in most cases it is due to bureaucracy associated with releasing payments, sometimes taking up to 9 months to honour requisition or pay an invoice yet we have a stock of LPA kits here in Nairobi", Kenyan Hain Life sciences officer. 
Table 2

The factors underlying lack of Xpert MTB/RIF and LPA services at district/county- and healthcare facilitylevel. The respondents were district (Uganda \& Tanzania) and/or county (Kenya) health officers and healthcare practitioners.

\begin{tabular}{|lllll|}
\hline & District/County level & \multicolumn{2}{l|}{$\begin{array}{l}\text { Healthcare facility } \\
\text { level }\end{array}$} \\
\hline $\begin{array}{l}\text { Limitation underlying absence of the } \\
\text { diagnostic test }\end{array}$ & $\begin{array}{l}\text { Xpert MTB/RIF }(\mathrm{N} \\
-76)\end{array}$ & $\begin{array}{l}\text { LPA } \\
(\mathrm{N}=\end{array}$ & $\begin{array}{l}\text { Xpert } \\
\text { MTB/RIF }\end{array}$ & $\begin{array}{c}\text { LPA } \\
(\mathrm{N}=\end{array}$ \\
\hline Under financing & & $201)$ & $(\mathrm{N}=34)$ & \begin{tabular}{c}
$138)$ \\
\hline Procurement difficulties
\end{tabular} \\
\hline Inadequate human resource & $91 \%$ & $55 \%$ & $56 \%$ & $21 \%$ \\
\hline No water & $9 \%$ & $7 \%$ & $6 \%$ & $8 \%$ \\
\hline No electricity & $1 \%$ & $3 \%$ & $24 \%$ & $0 \%$ \\
\hline Biosafety issues & $1 \%$ & $0 \%$ & $26 \%$ & $0 \%$ \\
\hline Only available at regional hospital & $0 \%$ & $1 \%$ & $26 \%$ & $0 \%$ \\
\hline Not aware of the test & $3 \%$ & $1 \%$ & $0 \%$ & $0 \%$ \\
\hline
\end{tabular}

Inadequate space and test complexity were also identified as reasons for underutilization of LPA. In principle conducting LPA requires three rooms (pre-PCR, PCR and post-PCR) to perform, "Very few healthcare facilities in the country have such space and person time to perform LPA", Tanzanian senior laboratory scientist.

\section{Culture}

Like LPA, culture was a less utilised diagnostic, $49 \cdot 6 \%$ (72/145) of the facilities that we audited. The median (range) of culture testing was $1(0-347)$ test per month. Whilst no culture test was prescribed by HCF level one, L2, L3, L4 and L5 prescribed 0.1 (0-0.2), 0.3 (0-36), $0.4(0-13)$ and 10 (0-347) cultures per month respectively. Utilization improved with HCF level, 0\% (0/6), 33\% (2/6), 40\% (23/57), 65\% (22/34) and $60 \%(25 / 42)$ level $1,2,3,4$ and 5 HCFs referring samples for culture. Where culture was prescribed, the percentage referral for drug sensitivity testing (DST) was $25 \%$ (18/72) overall and varied across HCF levels, $17 \%$ (1/6), 9\% (5/57), 6\% (2/34) and 24\% (10/42) at different HCF level 2-5 respectively. Time to culture restult was on average 2 month for all HCF (Fig. 6A and B).

\section{Monitoring response to anti-tuberculosis therapy}

Most of the 145 audited HCFs, 79\% (114) used microscopy as the only tool for monitoring treatment. This included 83\% (5/6), 50\% (3/6), 82 (47/57), 88\% (30/34) and 69\% (29/42) level 1, 2, 3, 4 and 5 HCFs respectively. Other treatment monitoring methods used alone were clinical assessment, 2\% (3) and Xpert 
MTB/RIF $0.7 \%$ (1). One of each level 2, 3 and 5 HCF used clinical assessment only while one level $3 \mathrm{HCF}$ used Xpert MTB/RIF. Some HCFs used a combination of monitoring methods, 3\% (4), 4\% (5), 4\% (5), 1.4\% (2) and $0.7 \%$ (1) for microscopy-clinical assessment, microscopy-Xpert MTB/RIF, Xpert MTB/RIF-culture and microscopy-Xpert MTB/RIF-chest X-ray respectively. Combination of microscopy and culture was available at two level 4- and three level 5- HCFs only. Microscopy-Xpert MTB/RIF was present at one level 1 and two of each level- 3 and 5 HCFs while microscopy-Xpert MTB-chest X-ray was available at one level 3 hospital.

\section{Human resource capacity}

We asked whether human resource capacity varied between HCF levels. All HCFs employed certificate and diploma level laboratory technicians and medically qualified staff with highest proportion (100\%) at level 1 and 2 HCFs respectively. There were no Bachelor degree qualified laboratory staff at level 1 HCFs. The highest proportion of master's degree holders, $38 \%$ and clerical staff, $75 \%$ was found at level $5 \mathrm{HCFs}$ (Table 3).

Table 3

Human resource capacity at different HCF levels. There is a trend to more highly qualified staff working in higher HCF levels.

\begin{tabular}{|c|c|c|c|c|c|c|}
\hline \multirow{3}{*}{$\begin{array}{l}\text { Human resource } \\
\text { capacity }\end{array}$} & \multirow{3}{*}{$\begin{array}{l}N \text { (median } \\
\text { (range) }\end{array}$} & \multicolumn{5}{|c|}{$\begin{array}{l}\text { Human resource proportional to number of that HCF } \\
\text { type }\end{array}$} \\
\hline & & L1 & L2 & L3 & L4 & L5 \\
\hline & & $\stackrel{(6}{\mathrm{HCFs})}$ & $\begin{array}{l}(6 \\
\text { HCFs })\end{array}$ & $\begin{array}{l}\text { (57 } \\
\text { HCFs) }\end{array}$ & $\begin{array}{l}\text { (34 } \\
\text { HCFs) }\end{array}$ & $\begin{array}{l}\text { (42 } \\
\text { HCFs) }\end{array}$ \\
\hline Certificate holders & $2(0-2)$ & $100 \%$ & $83 \%$ & $96 \%$ & $65 \%$ & $81 \%$ \\
\hline Diploma holders & $4(0-18)$ & $83 \%$ & $100 \%$ & $97 \%$ & $91 \%$ & $93 \%$ \\
\hline $\begin{array}{l}\text { Bachelor's degree } \\
\text { holders }\end{array}$ & $2(0-16)$ & $0 \%$ & $83 \%$ & $57 \%$ & $68 \%$ & $93 \%$ \\
\hline $\begin{array}{l}\text { Master's degree } \\
\text { holders }\end{array}$ & $0(0-6)$ & $0 \%$ & $17 \%$ & $0 \%$ & $15 \%$ & $38 \%$ \\
\hline Medically qualified & $5(0-60)$ & $100 \%$ & $67 \%$ & $83 \%$ & $82 \%$ & $74 \%$ \\
\hline Clerical staff & $1(0-35)$ & $17 \%$ & $33 \%$ & $63 \%$ & $38 \%$ & $57 \%$ \\
\hline
\end{tabular}

\section{Diagnostic choice making}

With a sub-set of healthcare practitioners $(\mathrm{N}=22)$, we explored the qualities they would consider to choose a diagnostic test for their practice. $95 \%$ and $82 \%$ of the practitioners preferred an expensive but more accurate and harder to perform but shorter time-to-result diagnostic to a low cost - less accurate and longer time-to-result test respectively (Table 4). 
Table 4

Qualities that inform choice of diagnostic test by practitioners. Accuracy and shorter time-to-result were considered more import than cost and ease of performing a test.

\begin{tabular}{|llc|}
\hline Diagnostic characteristic & No. of participants who made the choice & $\%$ \\
\hline Expensive but more accurate & 21 & 95 \\
\hline Cheap but less accurate & 1 & 5 \\
\hline Longer time-to-result (2 weeks) but easy to perform & 4 & 18 \\
\hline Shorter time-to-result $(4$ h) but hard to perform & 18 & 82 \\
\hline
\end{tabular}

\section{Policymakers' engagement}

Policy makers were engaged at the initiation-, during and after completion- of the study. Pre-study engagement gave policymakers' perspective on the important matters the study should focus on and the channels through which findings can be communicated to policy making organs. A total of 11 policymakers engagement workshops were held, three each in Kenya and Tanzania and five in Uganda. Dialogues ranged from ways to make research to policy making to sustainable uptake and utilization of diagnostic technologies for better health outcomes. Policy makers were keen to be involved in research right from inception to the end in order to increase translation of outputs into policy and practice. Demonstrating research outcomes and impact at county (region) level before scale-up to national level was particularly recommended as the best way to increase uptake of health research innovations. Creating a national health tax and permitting county-regional governments to invest a percent of local collected revenues was proposed as means of increasing domestic financing and ensure sustainable uptake and utilization of health technologies.

\section{Discussion}

Early diagnosis of tuberculosis is essential because it shortens time to appropriate treatment, prevents severe morbidity and mortality $(15,21)$. We set out to understand the barriers that hinder uptake of health research innovations and identify opportunities to maximise translation of these innovations into policy and practice in Low- and Middle- Income (LMIC) setting. Using implementation of tuberculosis diagnostics as a model, we found that barriers can be categorised into health system and socioeconomic - cultural barriers. In this paper we focus on health system challenges and show that uptake and utilization of molecular and microbiological (culture) diagnostics in LMIC setting is still below expectation. Low uptake of diagnostics was mainly attributed to underfinancing of healthcare followed by lack of awareness by both district health officers and practitioners at healthcare facilities.

Procurement difficulties, inadequate human resource and utilities such as water and electricity were highlighted as underlying sub-optimal utilization of molecular diagnostics hereby referred as Xpert MTB/RIF and LPA. 
Information given by district/county health officers through the online survey was corroborated by on-site $\mathrm{HCF}$ audits and interview of practitioners demonstrating the real situation on ground. While the private sector plays a major role in providing healthcare in the studied countries, government was found to be the key stakeholder providing over $80 \%$ of tuberculosis diagnostic and treatment services. This further emphasises the important position government occupies to identify and overcome underlying health system limitations and to maximise the uptake of health technologies ensuring they reach where they are needed most.

Despite its technical limitations, smear microscopy was widely implemented and utilized diagnostic for tuberculosis at all levels of the healthcare system in Kenya, Tanzania and Uganda $(22,23)$. We believe low- acquisition cost, space and energy requirements make the microscope more attractive for healthcare facilities to implement(24). Furthermore, the microscope is a multipurpose platform often serving other functions such as diagnosis of malaria, helminths and other bacterial infections in low income countries. Technical limitations such as low sensitivity and specificity for diagnosing tuberculosis have less impact on the choice of diagnostic methodology. Technical challenges notwithstanding, for example the low sensitivity of smear microscopy revealed by the 2016 Kenya TB prevalence survey where smear microscopy only detected $46 \%$ cases compared to $78 \%$ by Xpert MTB/RIF(25), a device that serves other diagnostic needs at the HCF makes more economic sense when deciding technologies to purchase. Therefore, investing in open rather than closed systems will go a long way in maximising uptake of new health technologies into policy and practice.

The World Health Organisation (WHO) approved Xpert MTB/RIF for implementation up to district hospital level ${ }^{9}$. Our results show that, out of the 57 level 3 (district/sub-county) hospitals, only $56 \%$ had the Xpert MTB/RIF instruments and only $14 \%$ utilized the instruments to capacity i.e., performing 8 or more tests per day. There was, instead, higher, close to $100 \%$ coverage at level 4 and 5 HCFs with corresponding high full capacity utilization of $27 \%$ and $51 \%$ respectively. It is therefore not clear the model followed in allocating the Xpert MTB/RIF instruments. For instance, in Kenya some counties had more than one Xpert MTB/RIF instruments per sub-county while others had two instruments serving over 10 sub-counties. In line with this observation Oliwa et al found variability in spatial distribution of diagnostic services in Kenya suggesting a non-need driven allocation of diagnostic platforms(26).

Only $2(1.4 \%)$ of the audited HCFs used the Xpert MTB/RIF at point-of-care (in consultation room). In most cases Xpert MTB/RIF results were not available in the same day, which means the speed advantage of Xpert MTB/RIF is not being realised. It is also not clear whether both doctor consultation and Xpert MTB/RIF testing were going on in the same room. The higher the HCF was in the health system hierarchy the higher the utilization of Xpert MTB/RIF instruments. Limitations such as procurement, inadequacy of utilities and human resources were less frequent at higher level HCFs. In line with Poorani et al, our findings show that a hub system with an effective sample referral network may be more cost-effective than placing Xpert MTB/RIF machine at every healthcare facility(27). With this approach, Xpert MTB/RIF may no longer qualify as near point-of-care test but its utilization will be more cost-effective. It has been suggested, however, that decentralisation of Xpert MTB/RIF can be cost-effective if the testing volumes 
are high in peripheral HCFs(28) and there is good flow of funds to ensure availability of consumables. The high testing volume - decentralisation model contradicts the novel battery powered single module point-of-care GeneXpert OMNI because it would require $16 \mathrm{~h}$ to test 8 samples thus increasing turnaround-time and cost more to implement in high TB burden settings $(29,30)$.

The Line probe Assay was the least implemented diagnostic test, yet it is the only approved rapid drug sensitivity testing (DST) diagnostic for multi-drug resistant TB. Although approved for implementation at regional (L4) hospital, only 1/34 (3\%) reported possession of LPA platform, which they were unfortunately unable to execute since they were trained by the test manufacturers. Lack of awareness emerged as the 2 nd main factor limiting the implementation of LPA. Surprisingly, almost $50 \%$ of health administrators and practitioners were not aware of LPA as a diagnostic for TB. Without awareness, there is no chance that such a diagnostic could be on the list of diagnostics to acquire by either HCF or district health administration. Space intensiveness and laboriousness were highlighted as limitations for high utilization by HCFs who had the LPA service. This is an eye opener for health technology developers to make technologies that are compatible with the available infrastructure as well as investing in increasing awareness and accessibility of these technologies especially in high TB burden settings.

Culture, the gold standard diagnostic for TB(31) was only found at a $5 \%$ implementation level across the three countries. All culture laboratories were associated with level $5 \mathrm{HCFs}$ (national, consultant or zonal referral hospitals). On average culture laboratories serve 20 million or more people. Level 5 hospitals were likely to perform culture and drug sensitivity testing (DST) than lower HCFs which did not have the facilities. Presence of a culture laboratory at a facility did not change the time-to-culture-result, 2 months at level 3, 4 and 5 HCFs. The limited availability of culture labs and very low referrals for culture shows the three countries are not on course to achieve the universal DST access target of $100 \%$ by 2020 (WHO framework of indicators and targets). While culture is relatively easy to perform and cheaper, practitioners indicated they would spend a little more money for a hard to perform, expensive but accurate test with shorter time-to-result diagnostic technology.

The low degree of coverage and utilization of molecular and culture tests revealed by our study is an indication that TB is most likely under-diagnosed in the region and more so, drug resistant TB. Global estimates show 3 million TB cases go undetected every year(7). Our findings also indicate that universal DST as recommended by WHO is currently unachievable until such a time when the countries have developed required diagnostic capabilities(32). Treatment response monitoring needs of drug susceptible TB in the region are largely met due to wide coverage of smear microscopy but not drug resistant TB which requires a combination of microscopy and culture to monitor $(33,34)$. Challenges associated with microscopy and culture for monitoring treatment response led the WHO to recognise the University of St Andrews developed tuberculosis Molecular Bacterial Load Assay (TB-MBLA) as a candidate to replace the two tests for monitoring TB treatment response $(35,36)$. Like other molecular diagnostics, maximising uptake and utilization of TB-MBLA requires addressing the barriers highlighted in this paper. 
In the same trend, human resources capacity increased with the level of HCF, which partly explains higher uptake and utilization of diagnostic services at these healthcare centres. Higher level HCFs are more likely to be in the urban centres with many social amenities attractive to professionals to come work and stay. Deliberate efforts must be made by national governments to ensure appointment and retention of healthcare practitioners in rural areas.

Health budgets in the study countries are largely donor dependent and thus any economic downturns in donor countries has ripple effect on the delivery of diagnostic and treatment services in LMIC settings. This is more so in the era of the COVID-19 pandemic that has ravaged global economy with traditional donor countries experiencing up to $10 \%$ or more shrinking of their economies(12). It is more prudent to state that LMICs probably need to address the need to increase domestic funding for healthcare including TB control services if the End TB Strategy has to be realised.

Engaging policymakers unravelled valuable ideas on how to practically increase research impact and domestic financing to sustain uptake of health technologies and ensure quality healthcare. A Health tax on particular goods and services to build revenue base for funding healthcare programmes, research and innovation was recommended as the best way to go. The views from policy makers clearly demonstrate how an invaluable partner they are in research and its translation to policy and practice. Response to COVID-19 is a good example where in most countries scientists and policy makers have worked hand-inhand to use available knowledge and tools to save lives whilst search for more effective medical remedies goes on.

\section{Conclusion}

Our results have revealed that the health system set up favours maximal uptake and utilization both molecular and microbiological diagnostics at tertiary rather than lower healthcare levels. They point to a hub-centralised model of implementation model as the most effective for maximising uptake and utilization of molecular diagnostics. It is important to note, however, that the success of a centralised system also requires unlocking the health system barriers to increase awareness, smoothen sample referral path and shorten results turn-around-time(37). Centralised system may mean low access to diagnostic services by people in rural and hard to reach areas, thus innovating ways to ensure access by such communities are crucial. The findings also show low diagnostic capacity for drug resistant tuberculosis in the region, which means they are far from achieving the universal DST recommended by WHO. This paper further emphasises that health system bottlenecks are multifactorial and thus call for multisectoral interdisciplinary approach to address them.

This paper follows the Squire style of reporting (see Additional file 3).

\section{List Of Abbreviations}


TB: Tuberculosis, HCF: Healthcare facility, WHO: World Health Organization, LPA: Line Probe Assay, DST: Drug Sensitivity Testing, MTB: Mycobacterium tuberculosis, RIF: Rifampicin.

\section{Declarations}

\section{Ethics approval and consent to participate}

The study gained ethical approval from respective institutional and national ethics committees represented in the consortium. In the UK, approval was obtained from University Teaching and Research Ethics Committee of University of St Andrews (MD12073), Uganda: Makerere University Institutional Research Ethics Board (IRB) and National Council of Science and Technology (HS 2129), Kenya: KEMRI Scientific and Ethics Review Unit (KEMRI/RES/7/3/1) and Tanzania: KCRI and Mbeya and southern highlands zonal IRBs and National Health Research Ethics Committee (NatHREC) at NIMR headquarters (NIMR/HQ/R.8a/vol.IX/1317). In addition to ethics approvals, access permissions were sought and obtained from local government authorities and healthcare facilities involved in the study.

\section{Consent for publication}

Not applicable.

\section{Availability of data and materials}

The quantitative data analyzed during the current study will not be made publicly available but are available from the corresponding author on reasonable request. The qualitative (transcripts) data analyzed during the current study are not publicly available due to them containing information that could compromise research participant privacy; the data collection tools are available on request.

\section{Competing interests}

The authors declare no conflict of interest.

\section{Funding}

The study was funded by the European and Developing Countries Clinical Trials Partnership (EDCTP), grant TWENDE-EDCTP-CSA-2014-283.

\section{Authors' contributions}

NEN, EA, MJ, SHG: Conceived and designed the study; WSFO, IM, AL, FN, KK, BM, EFM, ES, HM, DK, SM, BTM: Developed the research tools and collected the data; FO, IM, FN, WO, LM, NEN, WS: led database cleaning and data analysis; NEN \& WS: drafted the manuscript; WS \& AA: drew the figures and maps; All authors: reviewed the manuscript and SHG: proof read the manuscript.

\section{Acknowledgements}


The authors acknowledge the contributions of Dr Ewan Chirnside and Prof Gibson Kibiki in the initial study conception, design and implementation of the study. Prof Kibiki also played a big role in linking the TWENDE consortium to East African Health Research Commission (EAHRC). As a result of his effort, EAHRC officially adopted TWENDE as a research consortium for generating evidence needed for translation of health technologies to policy and practice in the East African Community. Special thanks to the healthcare administrators and practitioners, and policy makers who participated in the study.

\section{References}

1. World Health Organisation. WHO Coronavirus Disease (COVID-19) Dashboard [Internet]. WHO Health Emergency Dashboard. 2020 [cited 2020 Jul 25]. p. 1-8. Available from: https://covid19.who.int

2. WHO. Statement from Dr Mario Raviglione, WHO Director, Stop TB Department. Who [Internet]. 2012;11:26-8. Available from: https://www.who.int/tb/features_archive/mr_statement/en/

3. Nathavitharana RR, Friedland JS. A tale of two global emergencies: Tuberculosis control efforts can learn from the Ebola outbreak. Eur Respir J [Internet]. 2015;46(2):293-6. Available from: http://dx.doi.org/10.1183/13993003.00436-2015

4. World Health Organisation. Tuberculosis-Key facts. Geneva; 2020.

5. World Health Organization. Global Tuberculosis Report 2019 [Internet]. WHO Press. Geneva; 2019. Available from: https://www.who.int/tb/publications/global_report/en/

6. Houben RMGJ, Dodd PJ. The Global Burden of Latent Tuberculosis Infection: A Re-estimation Using Mathematical Modelling. PLoS Med. 2016;13(10):1-13.

7. Daniels B, Kwan A, Pai M, Das J. Lessons on the quality of tuberculosis diagnosis from standardized patients in China, India, Kenya, and South Africa. Vol. 16, Journal of Clinical Tuberculosis and Other Mycobacterial Diseases. 2019.

8. World Health Organisation. Molecular line probe assays for rapid screening of patients at risk of multi-drug resistant tuberculosis. Geneva; 2008.

9. World Health Organisation. Automated Real-time Nucleic Acid Amplification Technology for Rapid and Simultaneous Detection of Tuberculosis and Rifampicin Resistance: Xpert MTB / RIF System. Geneva; 2011.

10. Albert $H$, Nathavitharana RR, Isaacs $C$, Pai M, Denkinger CM, Boehme CC. Development, roll-out and impact of Xpert MTB/RIF for tuberculosis: What lessons have we learnt and how can we do better? Eur Respir J [Internet]. 2016;48(2):516-25. Available from:

http://dx.doi.org/10.1183/13993003.00543-2016

11. World Health Organization Regional Office for Europe. Rapid communication on the role of the GeneXpert $\circledast$ platform for rapid molecular testing for SARS-CoV-2 in the WHO European Region. 2020; (1 April 2020):1-8.

12. Hogan AB, Jewell BL, Sherrard-smith E, Vesga JF, Watson OJ, Whittaker $C$, et al. Potential impact of the COVID-19 pandemic on HIV , tuberculosis, and malaria in low-income and middle-income 
countries: a modelling study. Lancet Glob Heal [Internet]. 2020;(20):1-10. Available from: http://dx.doi.org/10.1016/S2214-109X(20)30288-6

13. World Health Organization. END TB Global Tuberculosis Report 2017. Geneva; 2017.

14. Sabiiti W, on behalf of the TWENDE Consortium. Beyond the Numbers: Interpreting WHO's Global Tuberculosis Report 2016 to Inform TB Policy and Practice in the East African Community [Internet]. Vol. 01, East African Health Research Journal. 2017. Available from: www.eahealth.org

15. World Health Organisation. End TB Strategy: global strategy and targets for tuberculosis prevention, care and control after 2015. World Heal Origanisation. 2013;53(9):1689-99.

16. Hays JP, Mitsakakis K, Luz S, van Belkum A, Becker K, van den Bruel A, et al. The successful uptake and sustainability of rapid infectious disease and antimicrobial resistance point-of-care testing requires a complex 'mix-and-match' implementation package. Eur J Clin Microbiol Infect Dis. 2019;38(6):1015-22.

17. Masini E, Hanson C, Ogoro J, Brown J, Ngari F, Mingkwan P, et al. Using Patient-Pathway Analysis to Inform a Differentiated Program Response to Tuberculosis: The Case of Kenya. J Infect Dis. 2017;216(June 2016):S714-23.

18. Hanson C, Osberg M, Brown J, Durham G, Chin DP. Finding the Missing Patients with Tuberculosis: Lessons Learned from Patient-Pathway Analyses in 5 Countries. J Infect Dis. 2017;216(August):S686-95.

19. Study C. Patient Pathway Analysis for DSD of TB in Kenya.

20. APA news. Tanzania enters middle income country status. APA news. 2020 Jul 2;1-2.

21. World Health Organisation. Early TB detection [Internet]. Geneva; 2018. Available from: https://www.who.int/tb/areas-of-work/laboratory/early-detection/en/

22. Desikan P. Sputum smear microscopy in tuberculosis: Is it still relevant? Tuberculosis. Indian J Med Res. 2013;137(3):442-4.

23. Sabiiti W, Mtafya B, Kuchaka D, Azam K, Viegas S, Mdolo A, et al. Optimising molecular diagnostic capacity for effective control of tuberculosis in high-burden settings. Int J Tuberc Lung Dis. 2016;20(8):1004-9.

24. Rieder HL, Van Deun A, Kam KM, Kim SJ, Chonde TM, Trébucq A, et al. Priorities for tuberculosis bacteriology services in low-income countries. Second edition. Paris: International Union Against Tuberculosis and Lung Disease, 2007. [Internet]. 2nd ed. luatld. Paris: International Union Against Tuberculosis and Lung Disease (The Union); 2007. 1-118 p. Available from: https://tbrieder.org/publications/books_english/red_book.pdf

25. Enos M, Sitienei J, Ong'ang'o J, Mungai B, Kamene M, Wambugu J, et al. Kenya tuberculosis prevalence survey 2016: Challenges and opportunities of ending TB in Kenya. PLoS One. 2018;13(12):1-19.

26. Oliwa JN, Maina J, Ayieko P, Gathara D, Kathure IA, Masini E, et al. Variability in distribution and use of tuberculosis diagnostic tests in Kenya: A cross-sectional survey. BMC Infect Dis. 2018;18(1). 
27. Pooran A, Theron G, Zijenah L, Chanda D, Clowes P, Mwenge L, et al. Point of care Xpert MTB/RIF versus smear microscopy for tuberculosis diagnosis in southern African primary care clinics: a multicentre economic evaluation. Lancet Glob Heal [Internet]. 2019;7(6):e798-807. Available from: http://dx.doi.org/10.1016/S2214-109X(19)30164-0

28. Sohn H, Kasaie P, Kendall E, Gomez GB, Vassall A, Pai M, et al. Informing decision-making for universal access to quality tuberculosis diagnosis in India: An economic-epidemiological model. BMC Med. 2019;17(1):1-11.

29. Schumacher SG. Xpert ultra and MTB/RIF same price. 2017;(June). Available from: https://www.teachepi.org/wp-content/uploads/Courses/ADV-2017/Xpert-MTB-RIF-Ultra-GeneXpertOMNI-status-update_18JUN2017.pdf

30. Ejalu DL. Cost-effectiveness analysis of genexpert omni compared to genexpert $\mathrm{mtb} / \mathrm{rif}$ for point of care diagnosis of tuberculosis among presumptive cases in pingire health centre iii, serere district. Vol. 10, MSPH. Makerere University; 2018.

31. Sabiiti W, Mtafya B, Kuchaka D, Azam K, Viegas S, Mdolo A, et al. Optimising molecular diagnostic capacity for effective control of tuberculosis in high-burden settings. Int $\mathrm{J}$ Tuberc Lung Dis. 2016;20(8).

32. de Vries SG, Cremers AL, Heuvelings CC, Greve PF, Visser BJ, Bélard S, et al. Barriers and facilitators to the uptake of tuberculosis diagnostic and treatment services by hard-to-reach populations in countries of low and medium tuberculosis incidence: a systematic review of qualitative literature. Lancet Infect Dis. 2017;17(5):e128-43.

33. World Health Organisation. Treatment of tuberculosis guidelines. 4th ed. Menzies R, Steingart $K$, Nunn A, Philips P, Schunemann H, McLellan F, et al., editors. Vol. WHO/HTM/TB, World Health Organisation. Geneva: World Health Organisation; 2010. 1-147 p.

34. World Health Organization (WHO). Companion handbook to the WHO guidelines for the programmatic management of drug-resistant tuberculosis. World Health Organization. Geneva; 2014. $464 \mathrm{p}$.

35. World Health Organization. Global tuberculosis report 2018 [Internet]. Geneva; 2018. Available from: http://apps.who.int/iris/bitstream/handle/10665/274453/9789241565646-eng.pdf?ua=1

36. Sabiiti W, Azam K, Farmer ECW, Kuchaka D, Mtafya B, Bowness R, et al. Tuberculosis bacillary load, an early marker of disease severity: The utility of tuberculosis Molecular Bacterial Load Assay. Thorax. 2020;1-3.

37. Nalugwa T, Shete PB, Nantale M, Farr K, Ojok C, Ochom E, et al. Challenges with scale-up of GeneXpert MTB/RIF ${ }^{\circledR}$ in Uganda: A health systems perspective. BMC Health Serv Res. 2020;20(1):17.

\section{Figures}



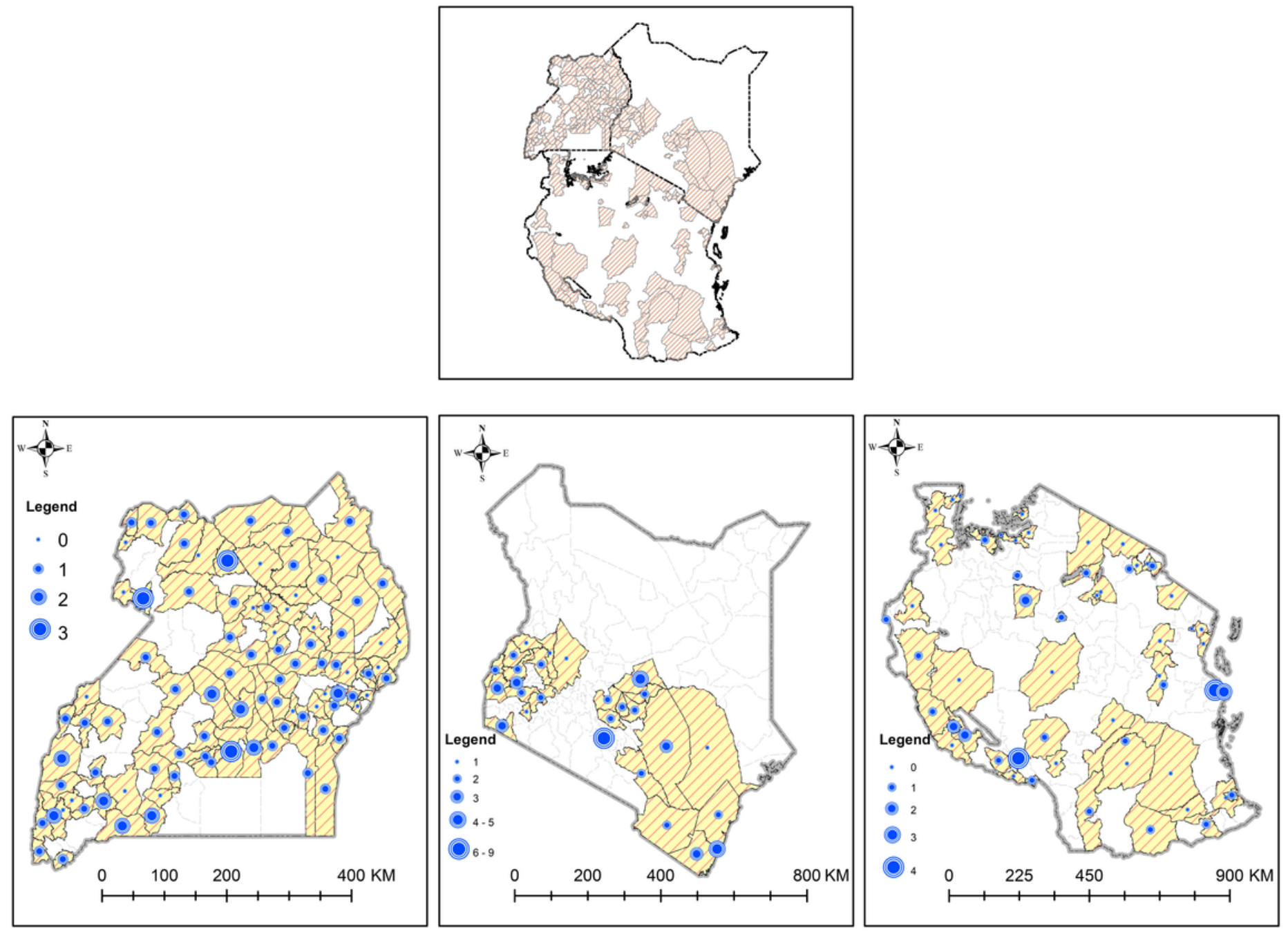

Figure 1

The Xpert MTB/RIF coverage in the surveyed districts and counties in Uganda, Kenya and Tanzania. The highest coverage was 3, 4 and 9 per district or county in Uganda, Tanzania and Kenya respectively. Orange strip shading denotes districts or counties surveyed. The size of the blue circles denotes the number of the Xpert MTB/RIF machines starting from zero or no Xpert MTB/RIF machine (smallest blue circle). 

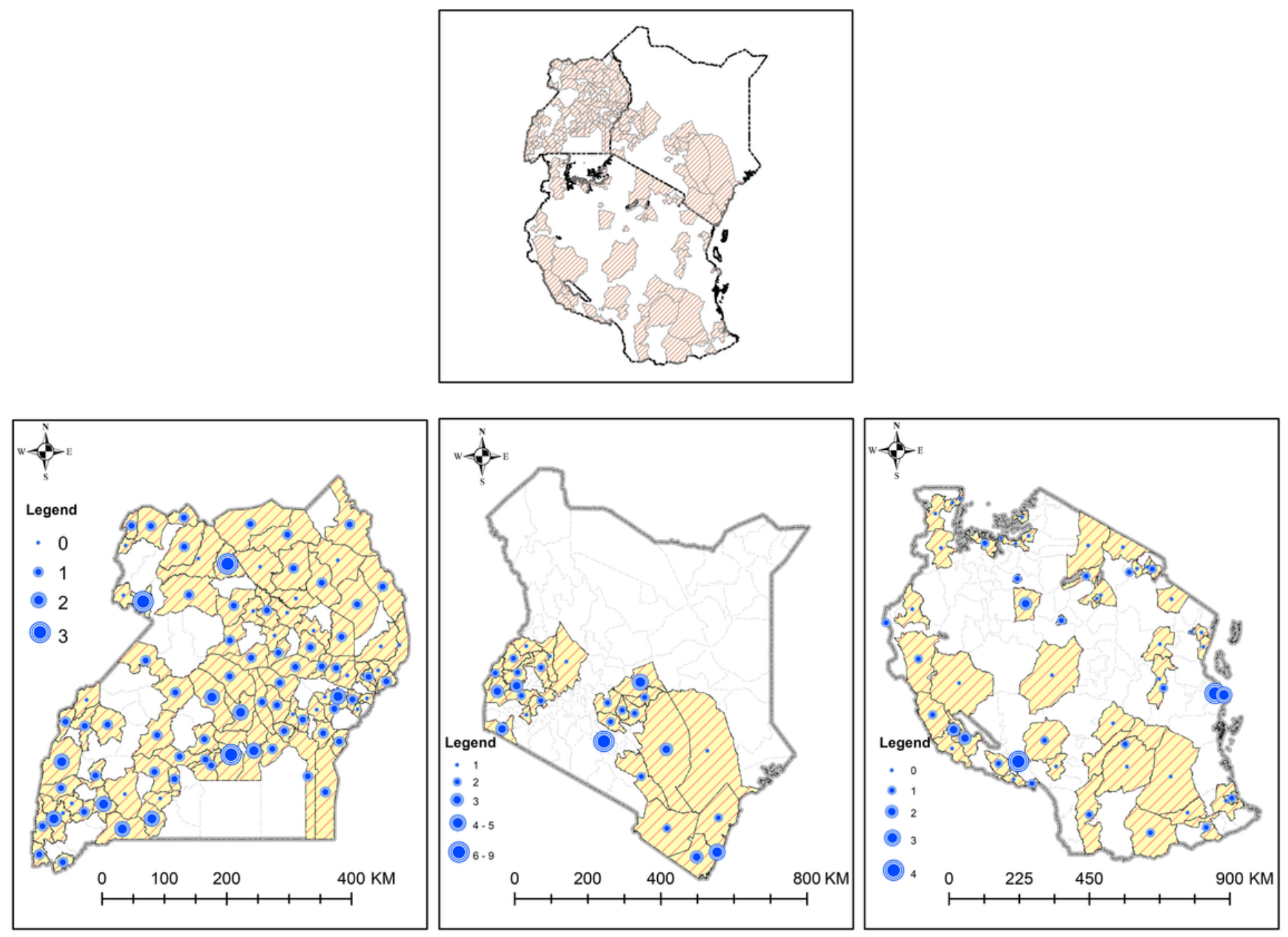

Figure 1

The Xpert MTB/RIF coverage in the surveyed districts and counties in Uganda, Kenya and Tanzania. The highest coverage was 3, 4 and 9 per district or county in Uganda, Tanzania and Kenya respectively. Orange strip shading denotes districts or counties surveyed. The size of the blue circles denotes the number of the Xpert MTB/RIF machines starting from zero or no Xpert MTB/RIF machine (smallest blue circle). 


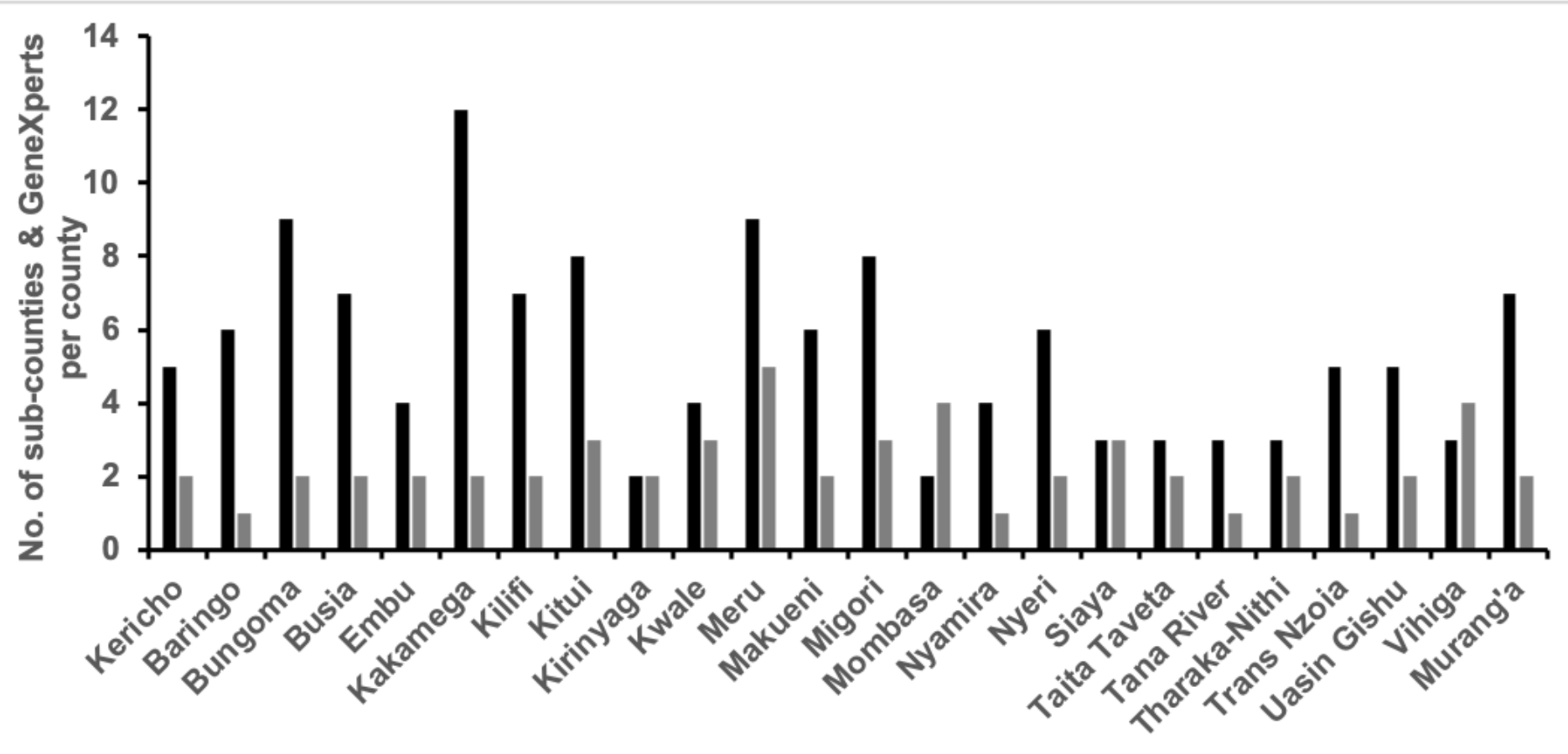

- No. of sub-Counties $\quad$ No. of GeneXpert machines

Figure 2

The sub-County level GeneXpert machine coverage in 27 Kenyan counties surveyed by TWENDE. There was no relationship between county size (number of sub-counties) and the number of GeneXpert machines allocated. Black bars $=$ Number of sub-counties, Grey bars $=$ Number of GeneXpert machines, $\mathrm{E}=$ Elgeyo.

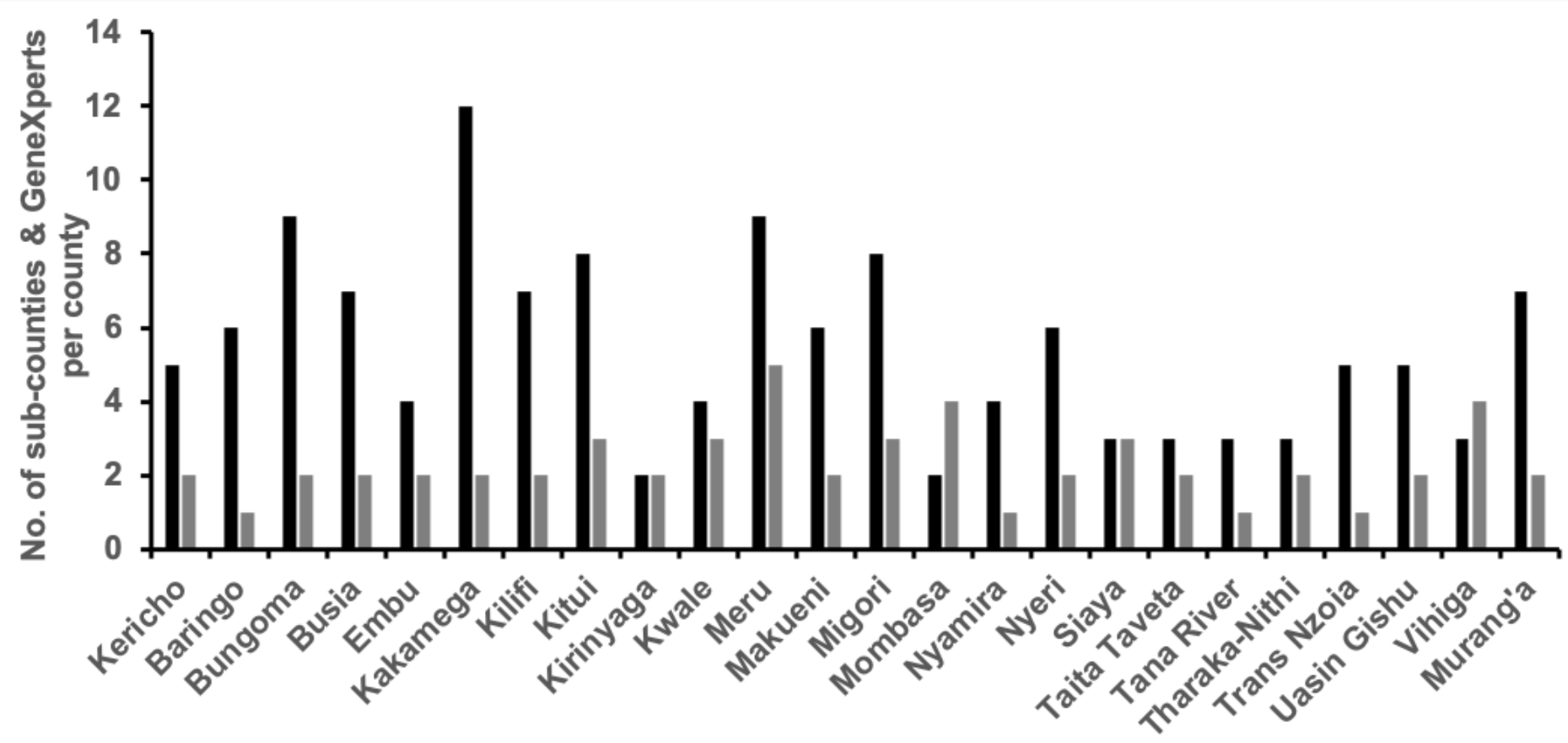

- No. of sub-Counties $\quad$ No. of GeneXpert machines 
Figure 2

The sub-County level GeneXpert machine coverage in 27 Kenyan counties surveyed by TWENDE. There was no relationship between county size (number of sub-counties) and the number of GeneXpert machines allocated. Black bars $=$ Number of sub-counties, Grey bars $=$ Number of GeneXpert machines, $\mathrm{E}=$ Elgeyo.

40.0

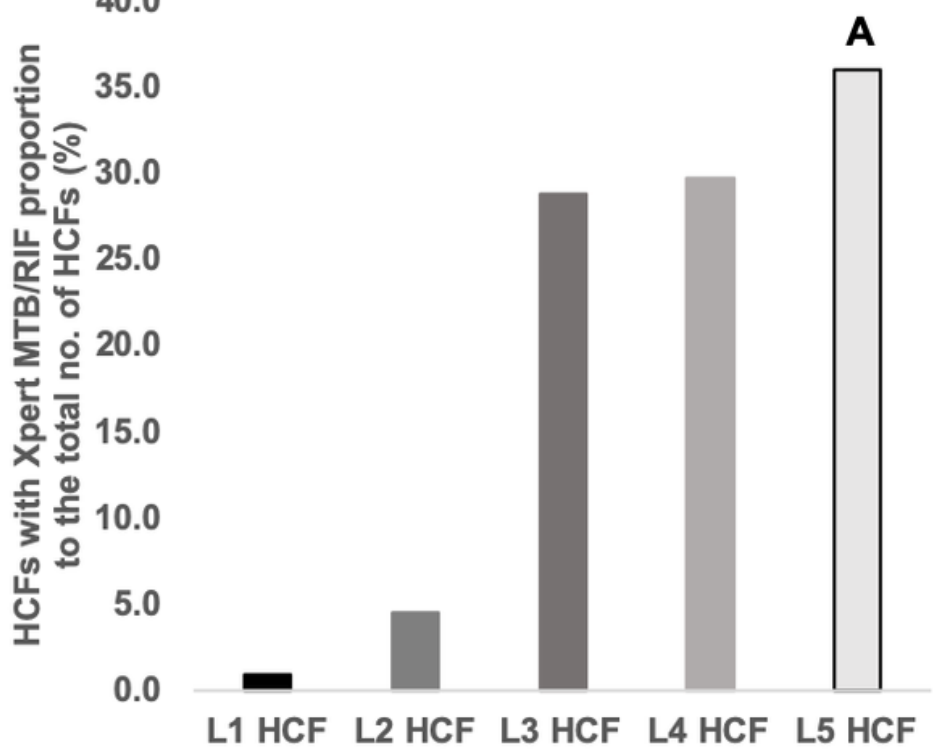

120.0

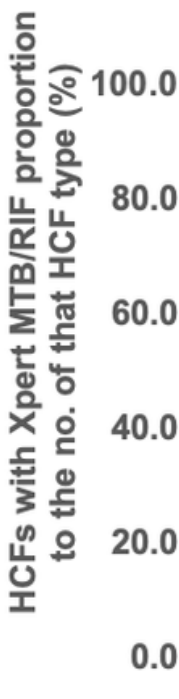

B

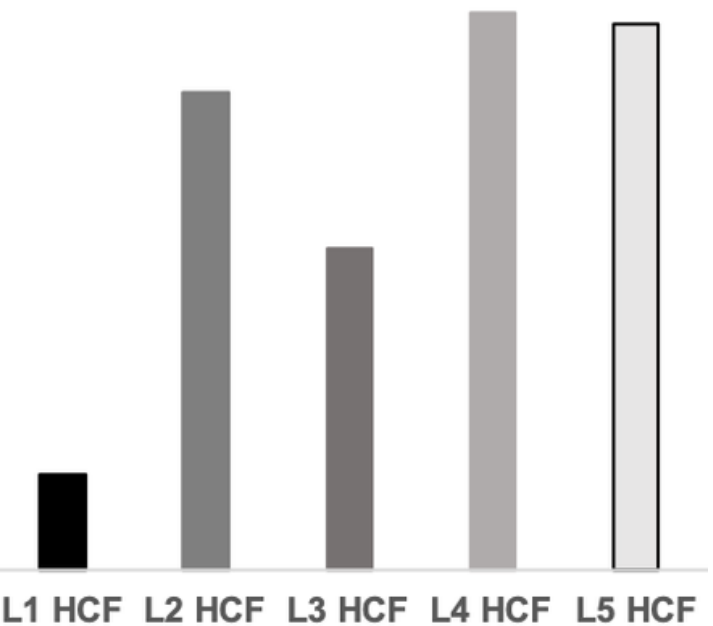

Figure 3

Xpert MTB/RIF coverage. A) percentage HCFs with Xpert MTB/RIF proportional to the total number of HCFs in possession of Xpert MTB/RIF (N=111). B) Xpert MTB/RIF coverage in proportion to the HCF type.

40.0

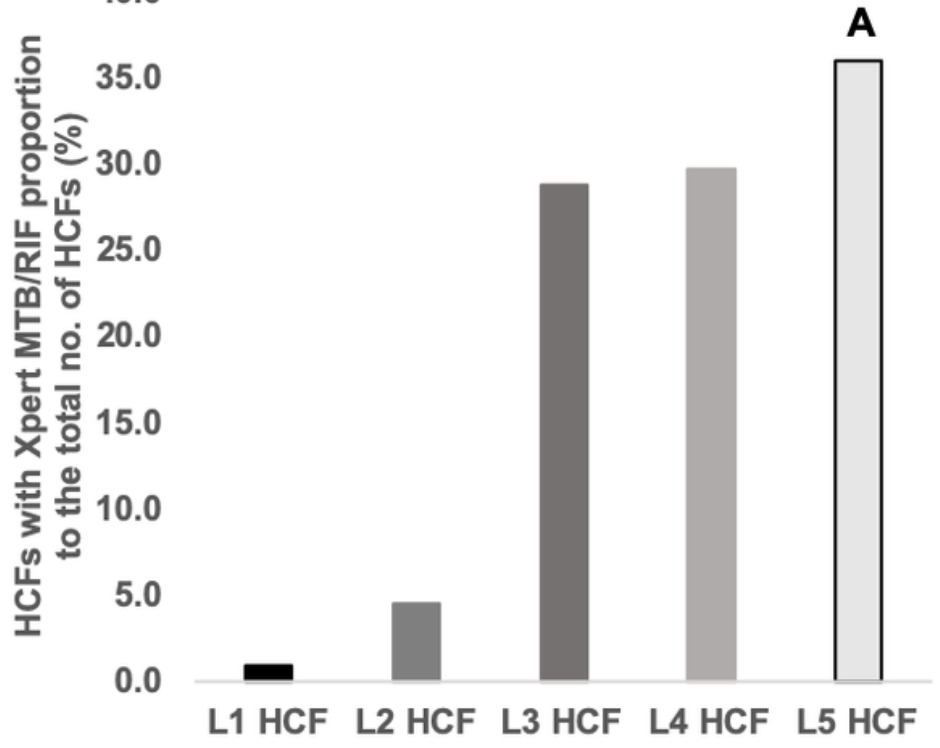

120.0

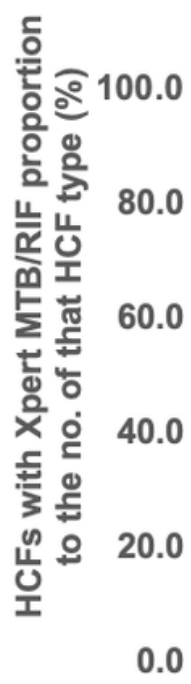

0.0
B

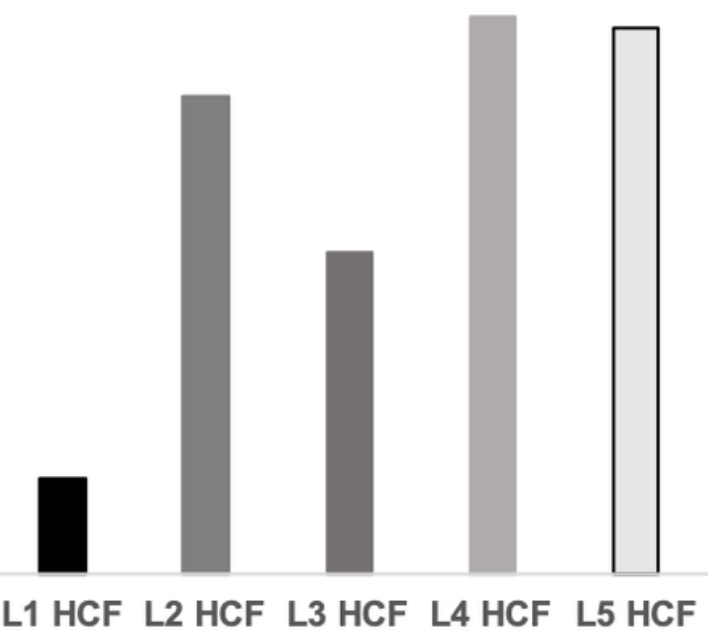

\section{Figure 3}

Xpert MTB/RIF coverage. A) percentage HCFs with Xpert MTB/RIF proportional to the total number of HCFs in possession of Xpert MTB/RIF ( $N=111)$. B) Xpert MTB/RIF coverage in proportion to the HCF type. 

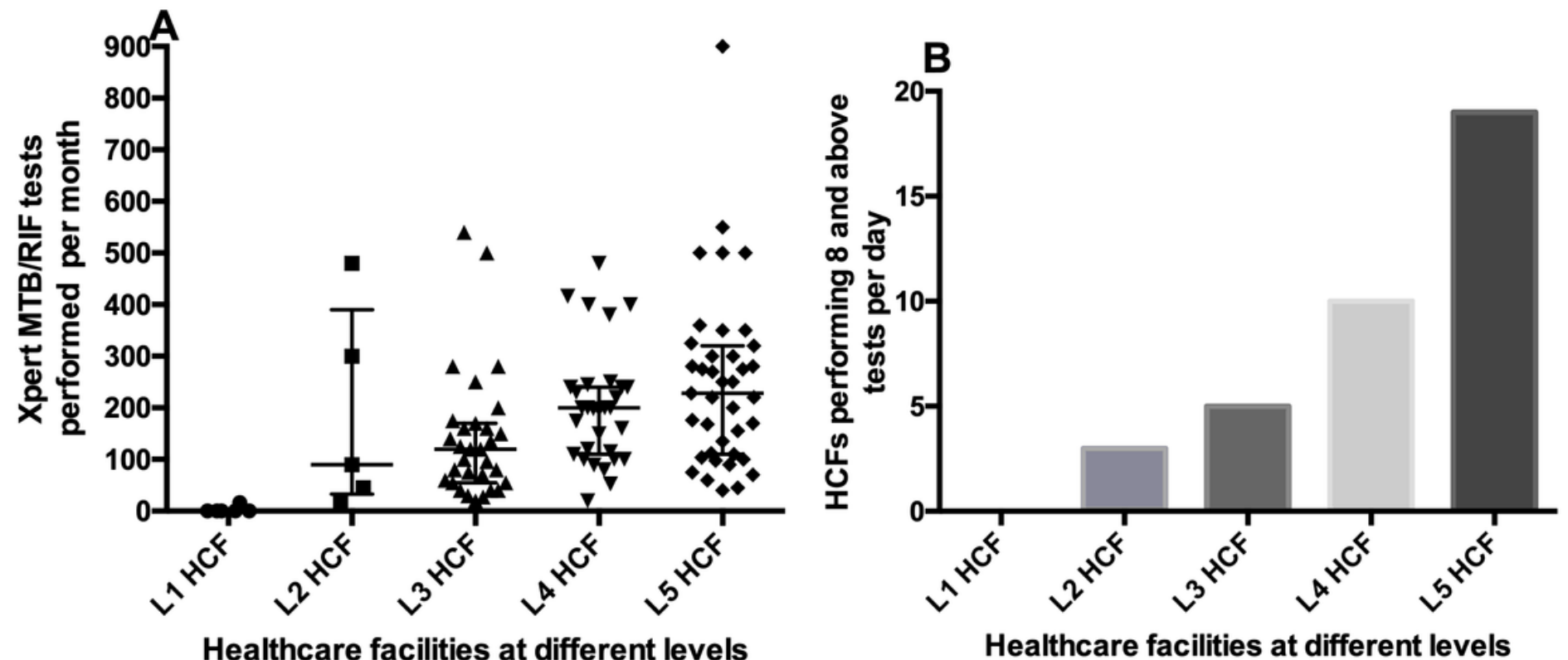

Healthcare facilities at different levels

Figure 4

Utilization of Xpert MTB/RIF at different HCF level. A) the number of Xpert MTB/RIF tests conducted per HCF. B) the number of HCFs that performed 240 and above tests per month or 8 tests per day recommended by WHO for full capacity use of Xpert MTB/RIF. In both level 5 hospitals performed more tests than lower level HCFs.
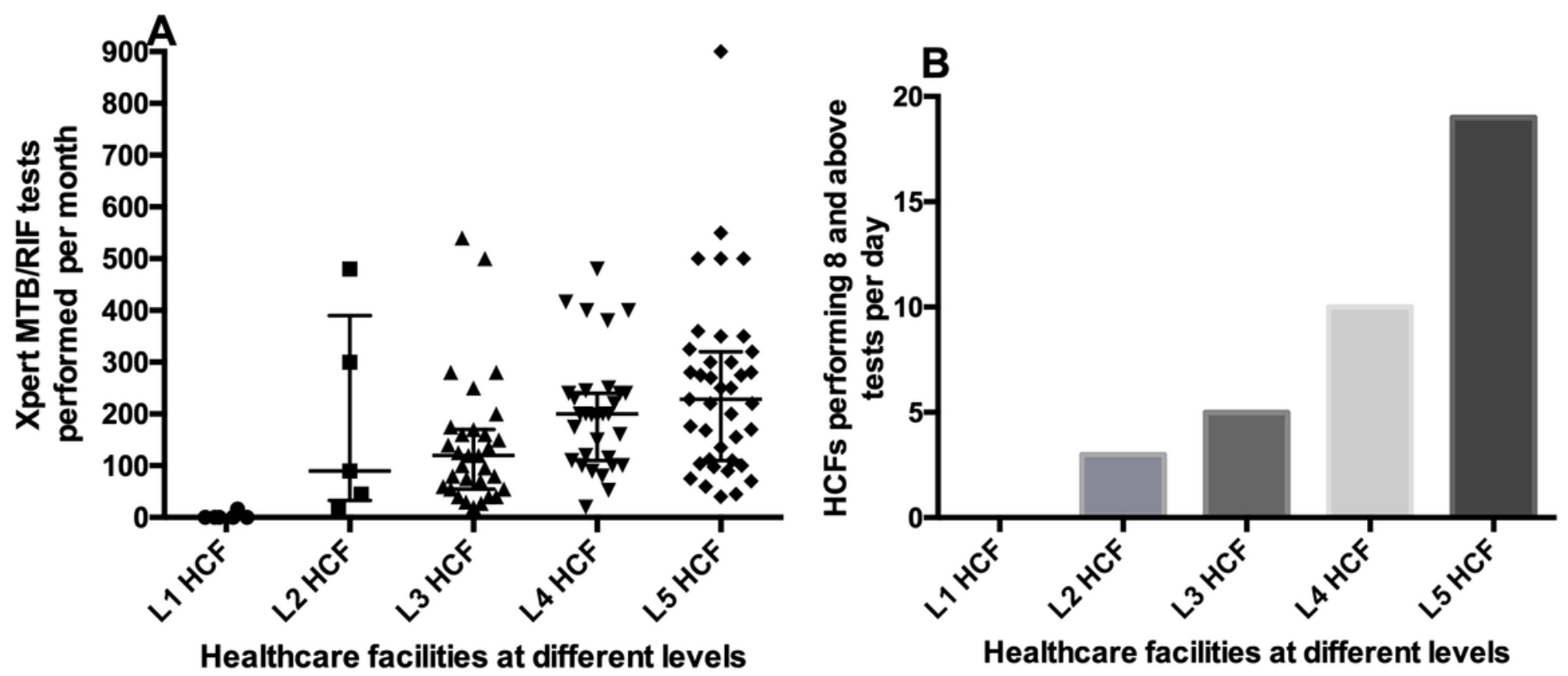

Figure 4

Utilization of Xpert MTB/RIF at different HCF level. A) the number of Xpert MTB/RIF tests conducted per HCF. B) the number of HCFs that performed 240 and above tests per month or 8 tests per day 
recommended by WHO for full capacity use of Xpert MTB/RIF. In both level 5 hospitals performed more tests than lower level HCFs.

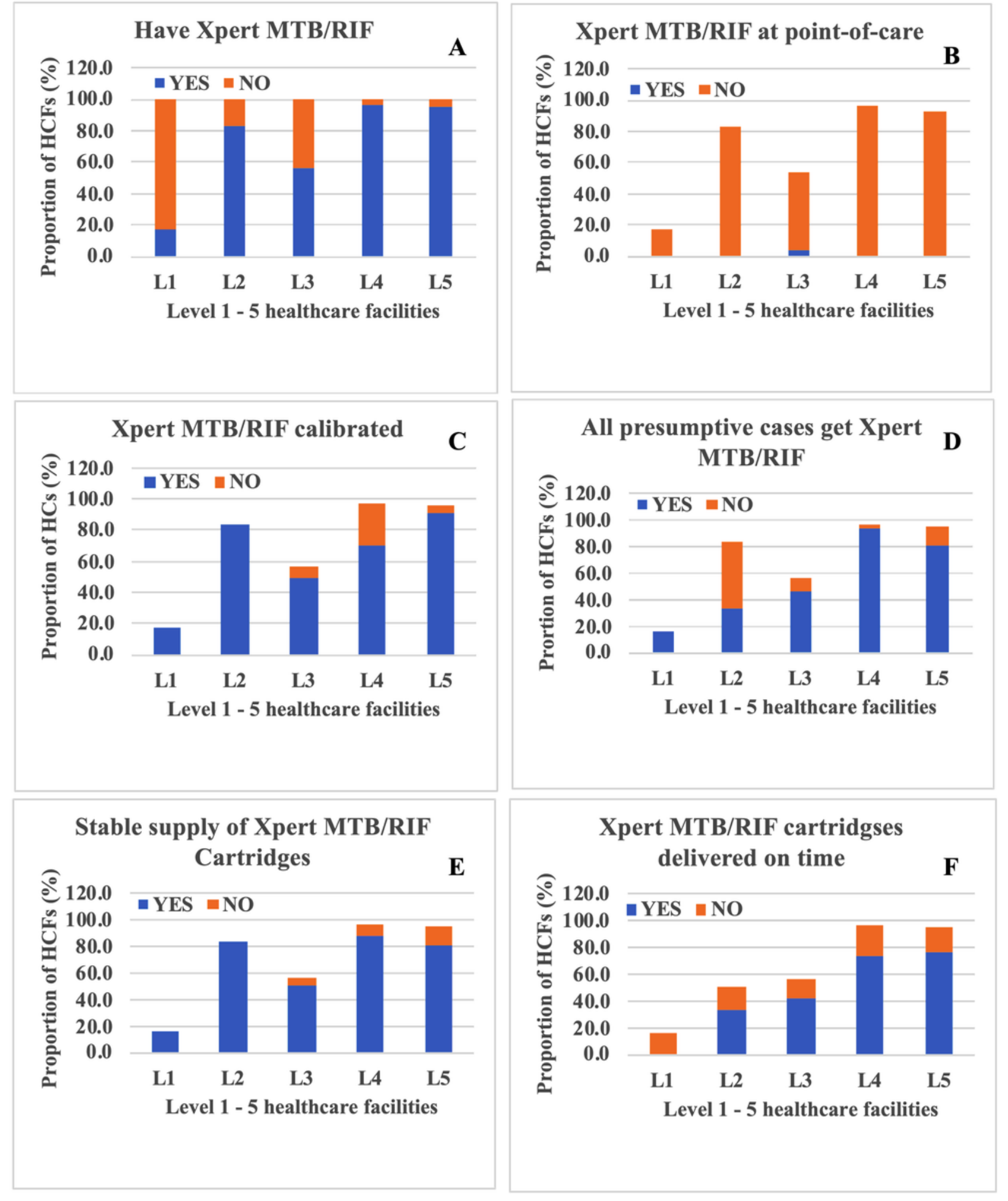

Figure 5

Availability, utilization, calibration and procurement issues of Xpert MTB/RIF machines. Participants answered yes (blue bars) or no (orange bars) to A) possession of Xpert MTB/RIF test instruments, B) use of the instrument at point-of-care, C) instrument calibration, D) prescription of Xpert MTB/RIF test to all 
presumptive MTB/RIF cases, E) stable supply of Xpert MTB/RIF reagent cartridges and F) if these cartridges were delivered on time. There was a trend to better performances in all parameters at higher healthcare facility levels.

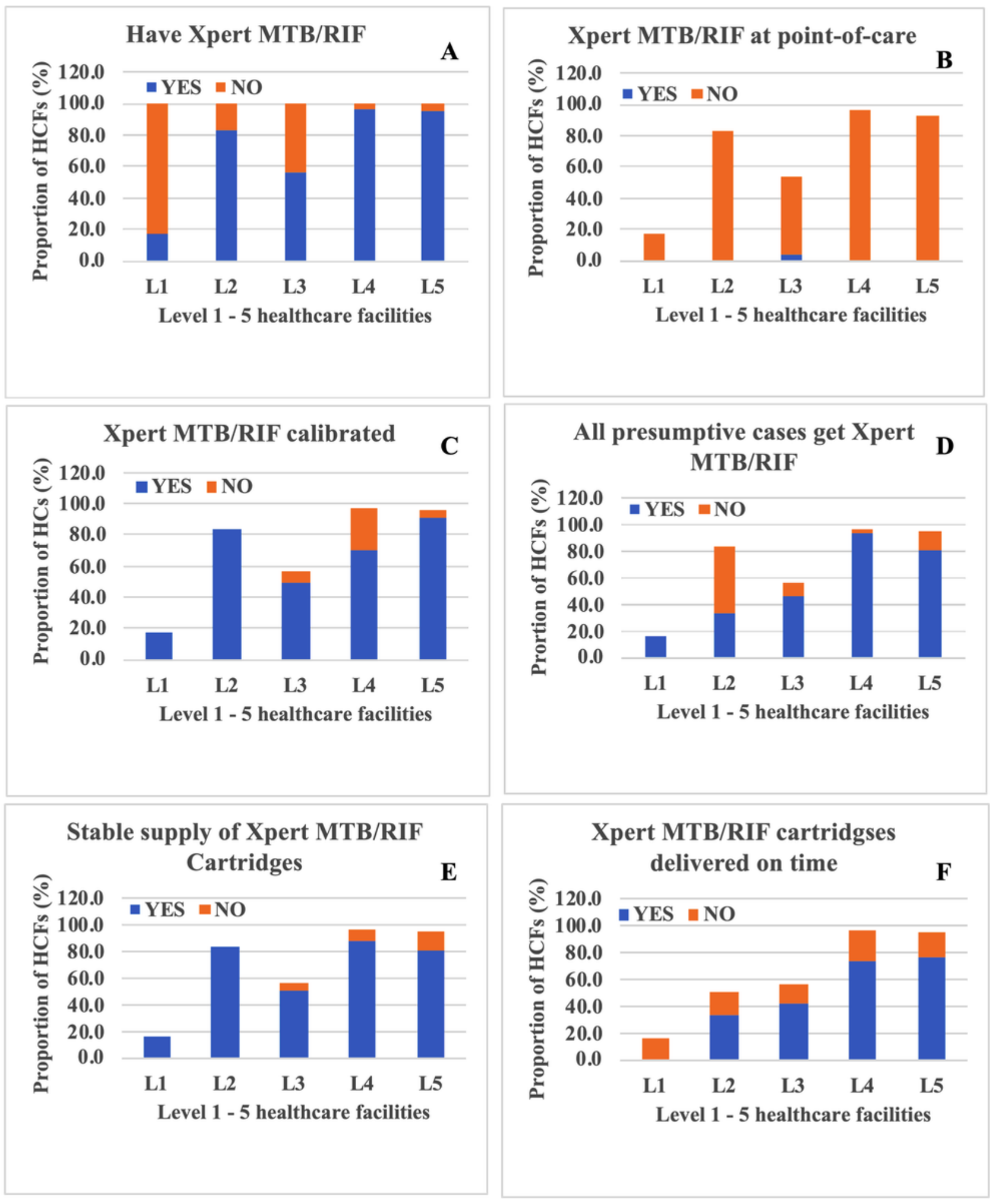

Figure 5

Availability, utilization, calibration and procurement issues of Xpert MTB/RIF machines. Participants answered yes (blue bars) or no (orange bars) to A) possession of Xpert MTB/RIF test instruments, B) use 
of the instrument at point-of-care, C) instrument calibration, D) prescription of Xpert MTB/RIF test to all presumptive MTB/RIF cases, E) stable supply of Xpert MTB/RIF reagent cartridges and F) if these cartridges were delivered on time. There was a trend to better performances in all parameters at higher healthcare facility levels.
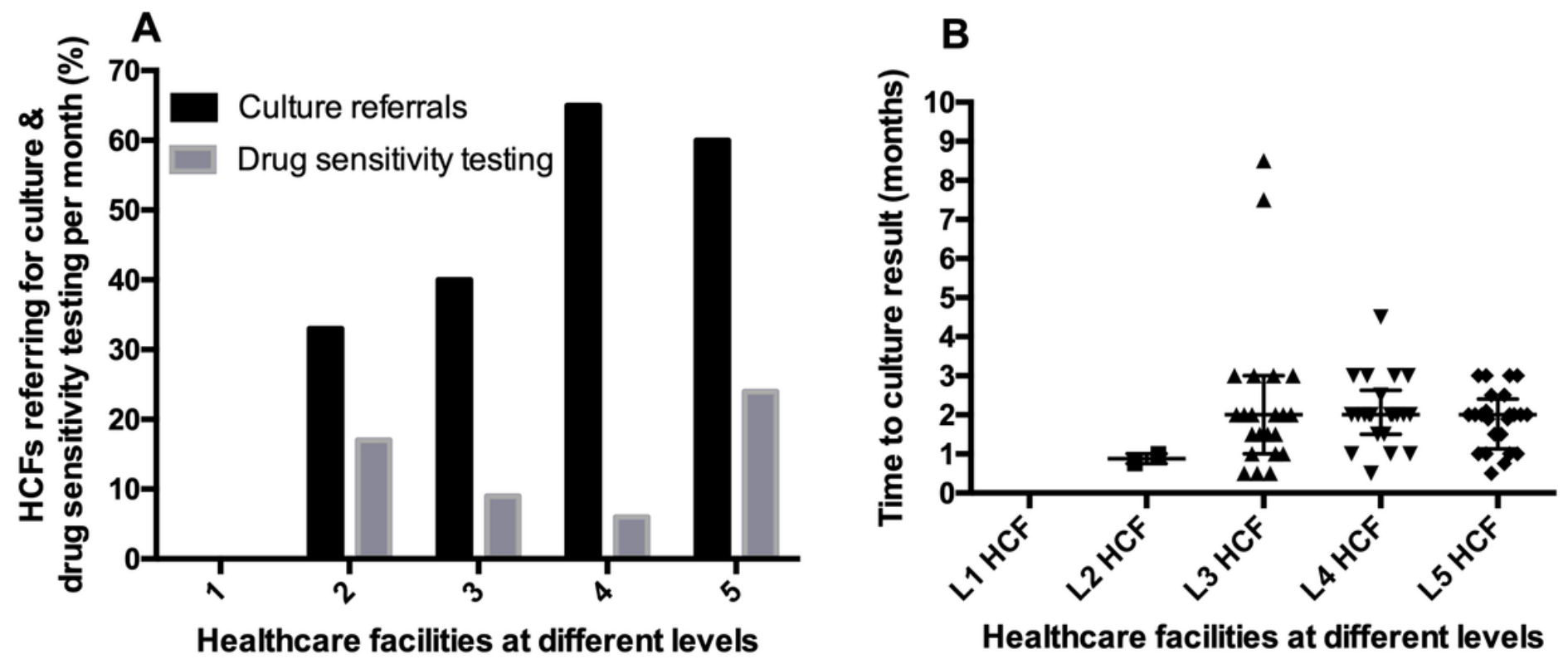

Figure 6

Healthcare facilities that referred samples for A) culture (black bars) and drug sensitivity testing (grey bars). B) Time to culture result across different healthcare facilities. Level $5 \mathrm{HCFs}$ performed substantially more DST than lower level HCFs. Note: the less median time-to-result by level 2 HCFs is because there were only 2 HCFs that referred samples for culture, insufficient to compute meaningful median.

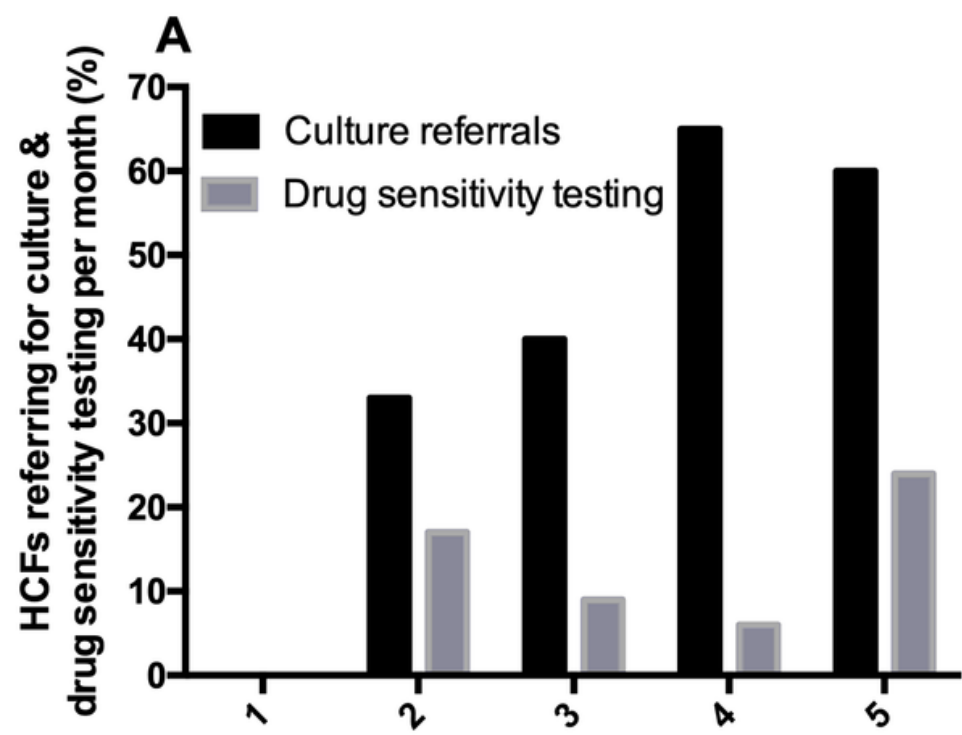

Healthcare facilities at different levels

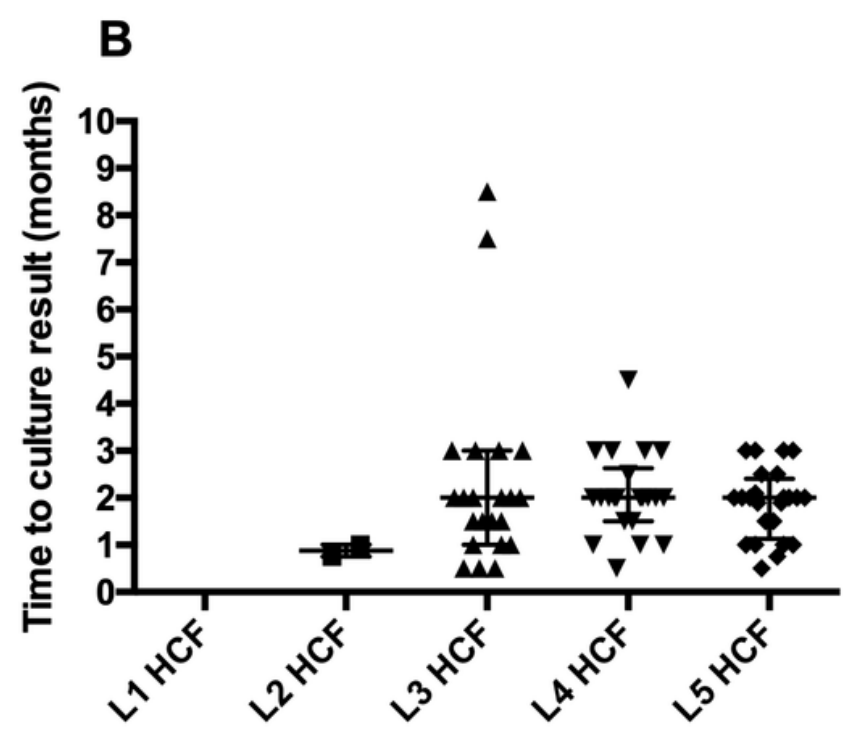

Healthcare facilities at different levels 


\section{Figure 6}

Healthcare facilities that referred samples for A) culture (black bars) and drug sensitivity testing (grey bars). B) Time to culture result across different healthcare facilities. Level $5 \mathrm{HCFs}$ performed substantially more DST than lower level HCFs. Note: the less median time-to-result by level 2 HCFs is because there were only $2 \mathrm{HCFs}$ that referred samples for culture, insufficient to compute meaningful median.

\section{Supplementary Files}

This is a list of supplementary files associated with this preprint. Click to download.

- Additionalfile1.docx

- Additionalfile1.docx

- Additionalfile2.docx

- Additionalfile2.docx

- Additionalfile3.pdf

- Additionalfile3.pdf 